\title{
Accidental diagnosis of a giant malignant peripheral nerve sheath tumor associated with neurofibromatosis type 1 in the back area: a case report
}

\author{
Dong Seok Shin, Woo Young Choi, \\ Ji Seon Cheon, Min Hyub Choi \\ Department of Plastic and Reconstructive \\ Surgery, Chosun University College of \\ Medicine, Gwangju, Korea
}

\begin{abstract}
Malignant peripheral nerve sheath tumors (MPNSTs) are rare and aggressive soft tissue sarcomas of neural origin. Approximately $50 \%$ of MPNSTs are associated with neurofibromatosis type $1(\mathrm{NF} 1)$, a neurocutaneous condition characterized by skin discoloration. MPNSTs occur in approximately $4 \%$ of patients with NF1. Malignancy should be suspected when a large mass consistent with a neurofibroma is observed with heterogeneity on a radiologic examination. In our case, immunohistochemistry revealed the presence of antigens for both the tumor protein $\mathrm{p} 53$ and the proliferation marker $\mathrm{Ki}-67$ (MKI67). In particular, MKI67 positivity helped to differentiate MPNST from neurofibroma. Complete surgical resection is the standard treatment. After surgery, radiation therapy is typically administered to the resection area to reduce the likelihood of recurrence. Following treatment, patients should undergo regular clinical follow-up using a combination of magnetic resonance imaging, computed tomography, and bone scanning for several years to monitor them for possible metastasis.
\end{abstract}

Keywords Malignant peripheral nerve sheath tumors / Neurofibromatosis / Case reports

\section{INTRODUCTION}

Malignant peripheral nerve sheath tumors (MPNSTs) are rare soft tissue sarcomas that occur in $8 \%-13 \%$ of patients with neurofibromatosis type 1 (NF1), a neurocutaneous condition characterized by skin discoloration. Meanwhile, in the general population, the incidence of MPNSTs has been estimated to be $0.001 \%[1,2]$. NF1 is one of the most important risk factors for MPNSTs [3]. MPNSTs often present as mass-like lesions of the head, neck, body, and other anatomical areas, with pain associated with neural system involvement [3].

Received: Aug 31, 2021 Revised: Nov 22, 2021 Accepted: Nov 24, 2021 Correspondence: Woo Young Choi Department of Plastic and Reconstructive Surgery, Chosun University College of Medicine, 365 Pilmundaero, Dong-gu, Gwangju 61453, Korea

Tel: +82-62-220-3180, Fax: +82-62-225-0996, E-mail: wychoi@chosun.ac.kr

Copyright $\odot 2022$ The Korean Society for Aesthetic Plastic Surgery.

This is an Open Access article distributed under the terms of the Creative Commons Attribution Non-Commercial License (https://creativecommons.org/licenses/by-nc/4.0/) which permits unrestricted non-commercial use, distribution, and reproduction in any medium, provided the original work is properly cited. www.e-aaps.org
MPNSTs are often confused with neurofibromas or plexiform neurofibromas in patients with NF1. As a result, there may be a delay in diagnosis and proper treatment [4]. Wide radical resection with negative margins is therapeutically effective, especially when followed by adjuvant radiotherapy [5]. Herein, we report a case of a patient with MPNST that was accidentally identified after two surgical resections, the specimens from which were diagnosed as neurofibroma, in a known NF1 patient.

\section{CASE REPORT}

A 55-year-old man with known NF1 visited our hospital. The patient had a mass measuring $12 \times 20 \mathrm{~cm}$ on his back and complained of discomfort in daily life. He had no history of radiation therapy. Physical examination revealed café-au-lait spots and multiple small masses across his entire body that were consistent with NF1; in addition, a large mass was visible on the left side of the back that was soft and tender upon palpation (Fig. 1). The patient had previously undergone surgery to remove the masses from the back on two occasions at the department of general surgery. The specimens from both operations were diagnosed as neurofibroma based on histo- 


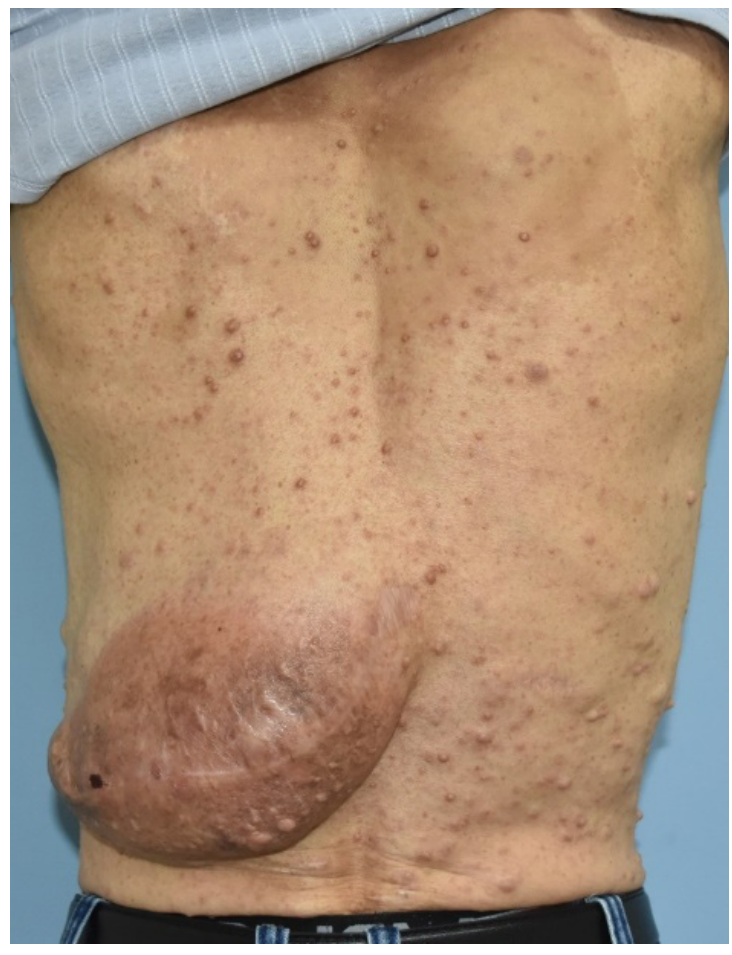

Fig. 1. Photograph of the patient's back. A mass measuring approximately $12 \times 20 \mathrm{~cm}$ was observed on the left side of the patient's back.

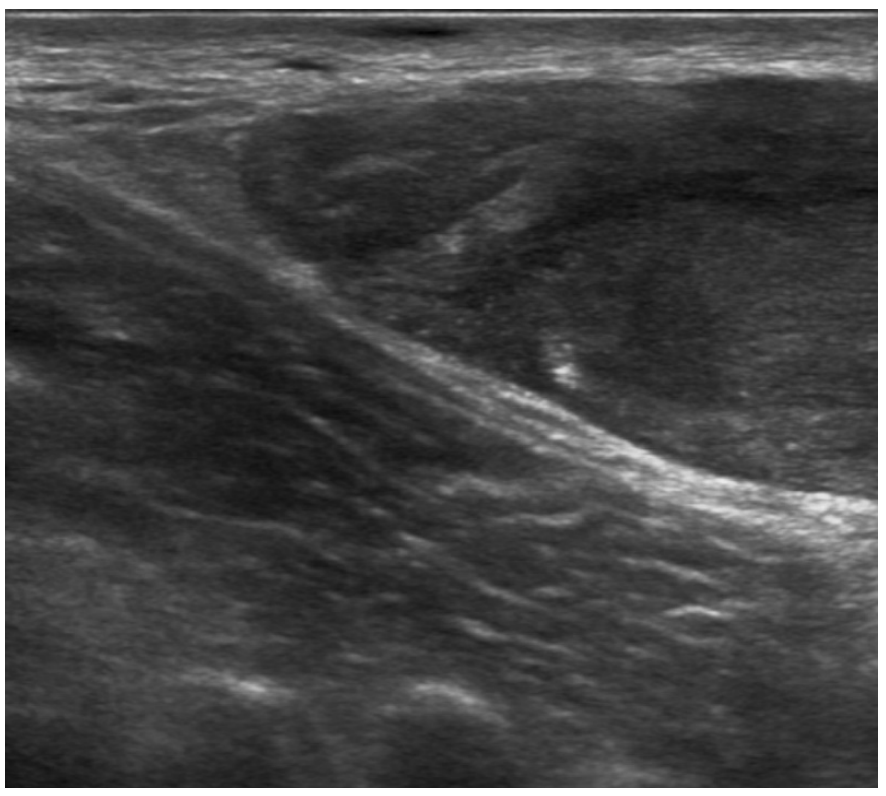

Fig. 2. Findings of an ultrasonographic examination. We detected a diffuse mass in the subcutaneous layer that was loculated, well-defined, and hypoechoic. There was no evidence of muscle invasion.

pathological and immunohistochemical evaluations by a pathologist. An ultrasonographic examination showed a well-defined, hypoechoic mass in the subcutaneous layer without any evidence of

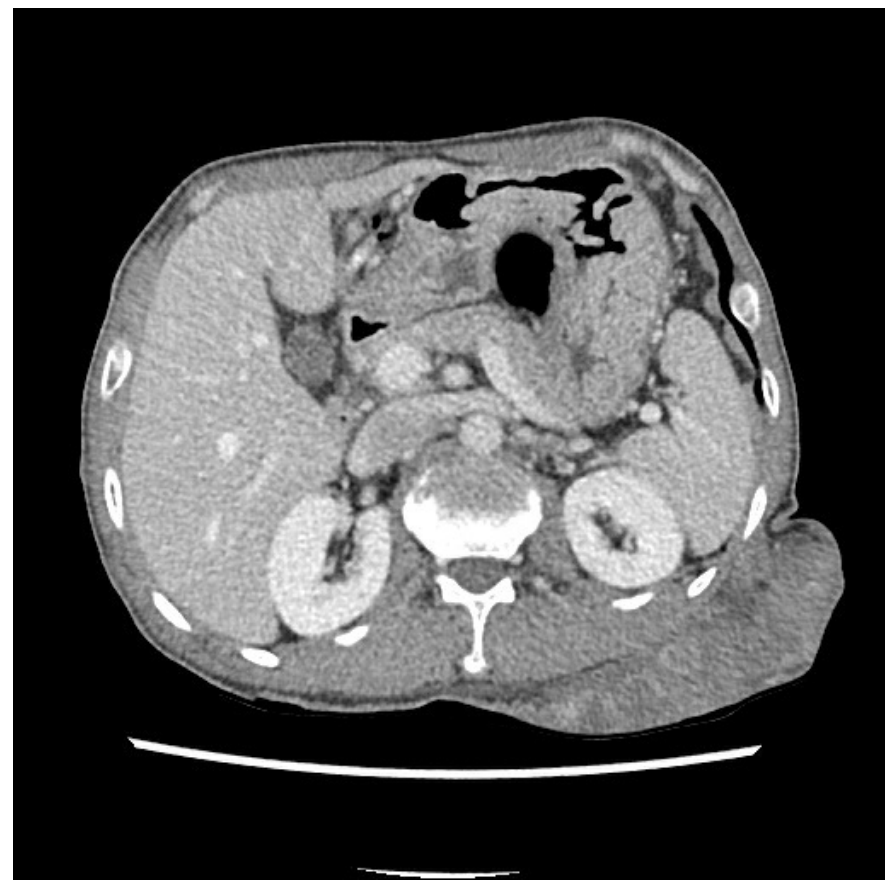

Fig. 3. Enhanced computed tomography of the abdomen. Large subcutaneous, heterogeneous masses were detected in the upper posterior abdominal wall, and multiple small neurofibromas were observed along the abdominopelvic wall.

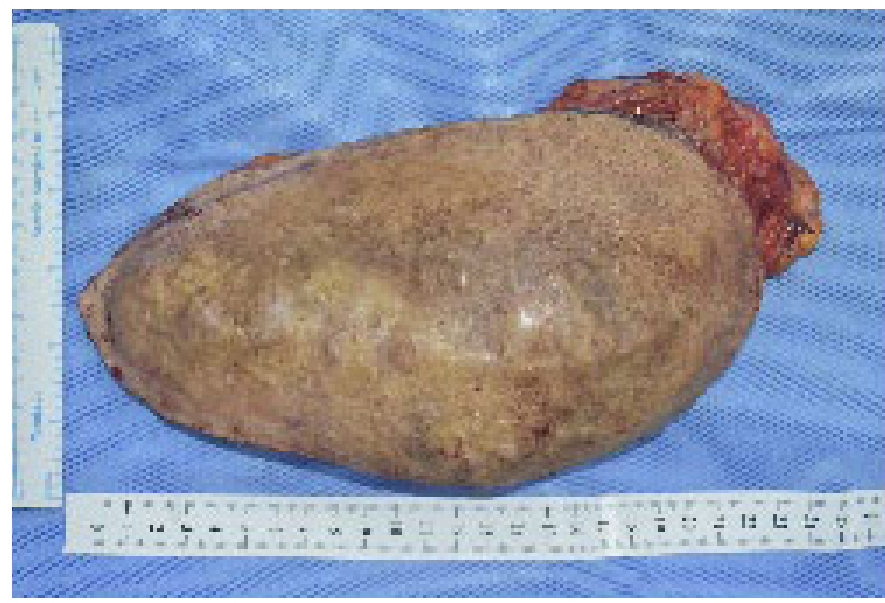

Fig. 4. Excised mass from the patient's back, measuring approximately $12 \times 20 \mathrm{~cm}$.

muscle invasion (Fig. 2). An enhanced computed tomography (CT) scan revealed a heterogeneous, subcutaneous, globular mass lesion in the upper posterior abdominal wall and confirmed the diagnosis of neurofibroma (Fig. 3).

The radiological evaluation and histopathological examination of specimens from the previous two operations had confirmed the diagnosis of neurofibroma. Hence, with the patient under general anesthesia, we performed total excision of the mass and skin grafting. A mass measuring approximately $12 \times 20 \mathrm{~cm}$ was excised from 

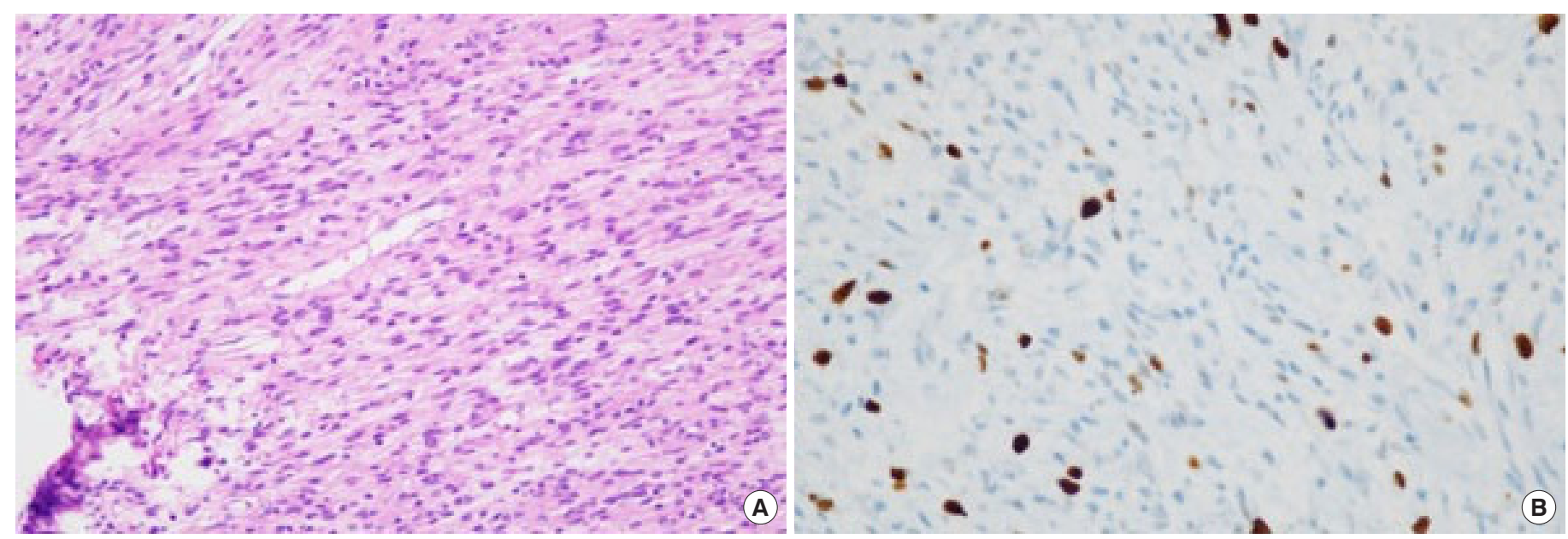

Fig. 5. Microphotographs of tissue samples. (A) Hematoxylin and eosin staining showing the typical herringbone pattern ( $\times 200)$. (B) Immunohistochemical staining showing darkly stained cancer nuclei. Positivity of the proliferation marker Ki-67 was observed in $20 \%$ of the cells ( $\times 200$ ).

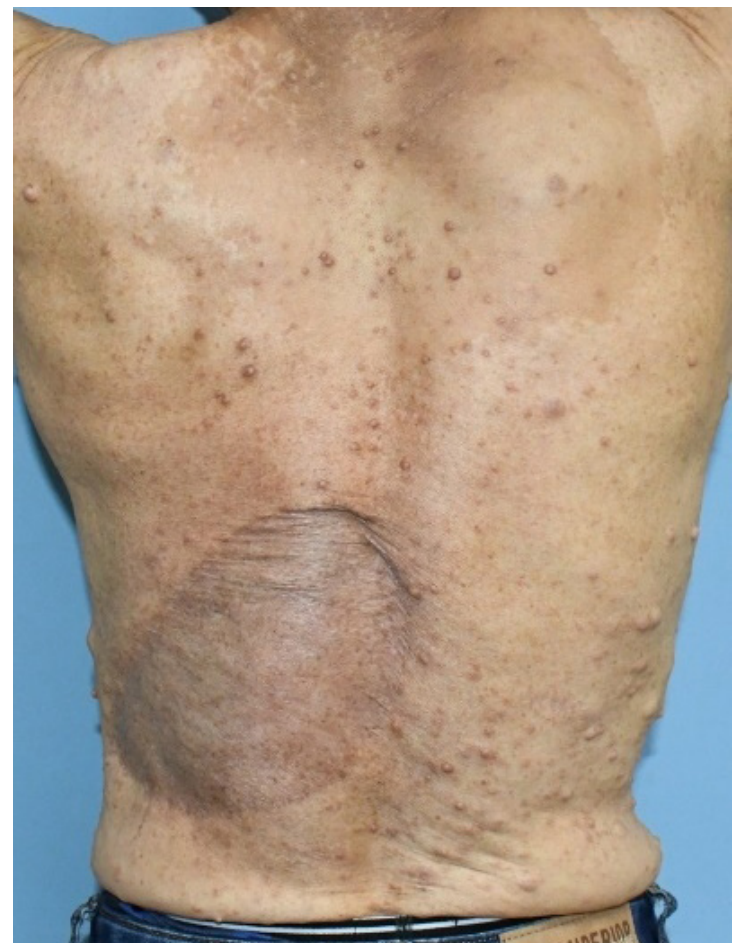

Fig. 6. Postoperative image of the patient's back. No recurrence or complication was detected after 18 months.

the patient's back, after which a split-thickness skin graft was performed (Fig. 4). The resected specimen was sent for microscopic examination, and immunohistochemical staining was performed. A typical herringbone pattern and diffuse atypical mitoses were observed in the histopathological examination, and immunohistochemical staining revealed positive expression (20\%) of the proliferation marker Ki-67 (MKI67) antigen (Fig. 5). The biopsies confirmed the diagnosis of MPNST associated with NF1. All resection margins were confirmed to be negative. In order to determine the extent of metastasis, the patient underwent positron emission tomography with fluorine-18-fluorodeoxyglucose. There was no significant hypermetabolic activity suggestive of metastasis. We advised the patient to undergo radiotherapy, which subsequently began 2 months after the operation. Daily adjuvant radiotherapy was administered at a dose of $180-200 \mathrm{cGy}$, amounting to a total dose of 50-55 Gy over a period of 6 weeks.

The patient was followed up in the outpatient department at 1,6, and 18 months. The patient's condition remained stable, and there were no postoperative complications at the operation site (Fig. 6).

\section{DISCUSSION}

MPNSTs are rare malignant soft tissue sarcomas that occur in the peripheral nerves, accounting for approximately $5 \%-10 \%$ of all soft tissue sarcomas [4,6]. Existing benign plexiform neurofibromas, a history of prior radiation treatment, large size, and mutation of the NF1 gene and surrounding genes are risk factors for the development of MPNSTs [7].

Early diagnosis of MPNSTs is important, and wide radical resection with negative margins is essential. However, distinguishing among MPNSTs, neurofibromas, and plexiform neurofibromas remains a clinical challenge. MPNSTs are often misdiagnosed in small samples such as those obtained by needle biopsy [8]. As a result, the diagnosis of MPNSTs is frequently delayed. Furthermore, the clinical features of MPNSTs, such as pain and neurological compromise, are similar to those of neurofibromas [2]. On ultrasonography, MPNSTs typically appear as well-defined, fusiform, hypoechoic masses [9]. CT can be used to determine the location and size of MPNSTs and to identify the presence of heterogeneous attenuation due to necrosis or hemorrhage. Ultrasonography and CT are rarely useful when differentiating between benign and malignant tumors [10]. Magnetic resonance imaging (MRI) may be use- 
ful for differentiating MPNSTs from neurofibromas. The presence of statistically significant MRI features is highly suspicious for malignancy. However, the diagnosis of MPNSTs should not rely solely on imaging; instead, all suspected cases should be biopsied [9,11].

Typically, radiological examinations of patients with MPNSTs reveal findings such as giant masses ( $>5 \mathrm{~cm}$ ), bony destruction, intratumoral lobulation, heterogeneous enhancement, perilesional edema, and local invasiveness $[9,10,12]$. If two or more of the aforementioned features are present, then an early diagnosis can be given [13]. Histologically, MPNSTs typically show a pattern of fasciculated growth of hyperchromatic spindle cells. Other common features include an elongated nucleus, perivascular hypercellularity, pronounced mitotic activity, and focal tumor necrosis [14]. Immunohistochemical studies using cell proliferation markers such as the MKI67 antigen have shown promise for predicting the prognosis of neoplasia. Moreover, the presence of the tumor protein p53, a tumor suppressor protein, has been reported in various soft tissues and neuronal neoplasms in which MKI67 was overexpressed [15].

In this report, we present a case where MPNST occurred following the diagnosis of neurofibroma, which was given based on biopsies performed after two separate operations. At first, we diagnosed the giant mass as a neurofibroma; however, it was diagnosed as MPNST following histopathological and immunohistological examinations of the resected specimen. Hence, we decided to perform adjuvant radiotherapy. Since MPNST is a life-threatening form of sarcoma, early diagnosis and wide radical excision are key to successful treatment. The possibility of malignant tumors should always be considered in NF1 patients presenting with very large masses.

\section{NOTES}

\section{Conflict of interest}

No potential conflict of interest relevant to this article was reported.

\section{Ethical approval}

The study was approved by the Institutional Review Board of Chosun University Hospital (IRB No. 2021-02-013).

\section{Patient consent}

The patient provided written informed consent for the publication and the use of his images.

\section{ORCID}

Dong Seok Shin

Woo Young Choi

Ji Seon Cheon
Min Hyub Choi

https://orcid.org/0000-0002-6844-0526

\section{REFERENCES}

1. Gupta A, Kumar R, Ojha BK, et al. Giant malignant peripheral nerve sheath tumor of scalp in non-neurofibromatosis person: a rare case report. Indian J Neurosurg 2018;7:54-7.

2. Widemann BC. Current status of sporadic and neurofibromatosis type 1-associated malignant peripheral nerve sheath tumors. Curr Oncol Rep 2009;11:322-8.

3. Pourtsidis A, Doganis D, Baka M, et al. Malignant peripheral nerve sheath tumors in children with neurofibromatosis type 1. Case Rep Oncol Med 2014;2014:843749.

4. Ducatman BS, Scheithauer BW, Piepgras DG, et al. Malignant peripheral nerve sheath tumors: a clinicopathologic study of 120 cases. Cancer 1986;57:2006-21.

5. Kumar P, Jaiswal S, Agrawal T, et al. Malignant peripheral nerve sheath tumor of the occipital region: case report. Neurosurgery 2007;61:E1334-5.

6. Carli M, Ferrari A, Mattke A, et al. Pediatric malignant peripheral nerve sheath tumor: the Italian and German soft tissue sarcoma cooperative group. J Clin Oncol 2005;23:8422-30.

7. Prudner BC, Ball T, Rathore R, et al. Diagnosis and management of malignant peripheral nerve sheath tumors: current practice and future perspectives. Neurooncol Adv 2019;2(Suppl 1):i40-9.

8. Amirian ES, Goodman JC, New P, et al. Pediatric and adult malignant peripheral nerve sheath tumors: an analysis of data from the surveillance, epidemiology, and end results program. J Neurooncol 2014;116: 609-16.

9. Kamran SC, Shinagare AB, Howard SA, et al. A-Z of malignant peripheral nerve sheath tumors. Cancer Imaging 2012;12:475-83.

10. Gladish GW, Sabloff BM, Munden RF, et al. Primary thoracic sarcomas. Radiographics 2002;22:621-37.

11. Wasa J, Nishida Y, Tsukushi S, et al. MRI features in the differentiation of malignant peripheral nerve sheath tumors and neurofibromas. AJR Am J Roentgenol 2010;194:1568-74.

12. Kar M, Deo SV, Shukla NK, et al. Malignant peripheral nerve sheath tumors (MPNST): clinicopathological study and treatment outcome of twenty-four cases. World J Surg Oncol 2006;4:55.

13. Yu YH, Wu JT, Ye J, et al. Radiological findings of malignant peripheral nerve sheath tumor: reports of six cases and review of literature. World J Surg Oncol 2016;14:142.

14. Gogate BP, Anand M, Deshmukh SD, et al. Malignant peripheral nerve sheath tumor of facial nerve: presenting as parotid mass. J Oral Maxillofac Pathol 2013;17:129-31.

15. Watanabe T, Oda Y, Tamiya S, et al. Malignant peripheral nerve sheath tumours: high Ki67 labelling index is the significant prognostic indicator. Histopathology 2001;39:187-97. 